7. Elektrische DurchbruchFeldstärke von Gasen, W. O. Schumann. Julius Springer (Berlin), 1925. Curve of dielectric strength of air, p. 25.

8. Irving Langmuir, A.I.E.E. Trans., v. 32, 1913, p. 1921, and Am. Chem. Soc. Jl., v. 38, 1916, p. 2283; also Am. Chem. Soc. Jl., v. 40, 1918, p. 1387

9. C. J. Davisson and L. H. Germer, Phys. Rev., v, 30, no. 6, 1927, p. 705; also Zeit. für Phys., v. 54, 1929, p. 408.

10. Puncture and Arcover in Air at Pressures of 1 to 30 Atmospheres, Carl Reher. Archiv. für Elektrotech., v. 25, 1931, p. 277.

11. Arcover of Solid Insulators in Air, L. Inge and A. Walther. Archiv. für Elektrotech., v. 26, 1932, p. 409.

12. An Optical Study of Spark Breakdown, F. G. Dunnington. Phys. Rev., v. 38, 1931, p. 1535 .

13. An Experimental Solution of Electrical Problems, E. W. Rice. A.I.E.E. Trans., v. 36,1917 , p. 905.
14. A Vacuum Discharge Tube por 2.4 Megavolts, A. Brasch and F. Lange Naturwiss., v. 18, 1930, p. 765.

15. Dielectric Phenomena in High Voltage Engineering, F. W. Peek, Jr. McGraw-Hill, 1929, third edition.

16. The Effect of Humidity on Dry Flashover, J. T. Littleton and W. W. Shaver, A.I.E.E. Trans., v. 47,1928 , p. 438.

17. Normal Frequency Arcover Values of Insulators, H. A. Frey and K. A. Hawley. A.I.E.E. Trans., v. 51, 1932, p. 690

18. Insulator Sparkover, W. L. Lloyd, Jr. A.I.E.E. Trans., v. 51, 1932, p. 669 .

19. Suspenston Insulators, Economic Selection, J. J. Torck and C. G. Archibald. A.I.E.E. Trans., v. 51, 1932, p. 669.

20. The Sixty-Cycle Flashovek of long Suspension Insulator Strings, R. H. Angus. A.I.E.E. Trans., v. 49, 1930, p. 15.

\title{
A Glow Discharge Anemometer
}

\begin{abstract}
A glow discharge at atmospheric pressure is responsive to air velocity, and may be used as an anemometer. The properties of such a glow are discussed in this paper, and data relating to glow current, voltage, glow length, and air velocity are presented. It is also shown that the glow discharge responds to rapid fluctuations in air velocity and is therefore a practical device for investigating turbulence. A comparison of such an anemometer with the previously used hot wire method is given.
\end{abstract}

\section{By \\ FREDERICK C. LINDVALL ASSOCIATE A.I.E.E.}

Californid Institute of

$\mathbf{R}$

ECENT aerodynamical research of both theoretical and experimental nature has indicated the important influence which air turbulence has on wind tunnel measurements. Experimental quantities, the lift and drag coefficients, are materially affected by the existence of small fluctuations superposed on the measured stream velocity. Moreover, these fluctuations in velocity, constituting turbulence, are characteristically of relatively high

Full text of a paper recommended for publication by the A.I.E.E. committee on research, and scheduled for discussion at the A.I.E.E. Pacific Coast convention, Salt Lake City, Utah, Sept. 3-7, 1934. Manuscript submitted April 23 1934; released for publication June 4, 1934. Not published in pamphlet form. 1. For all numbered references see list at end of paper. frequency and random nature, so that their presence is not indicated by air speed meters, manometers, or other velocity measuring devices involving inertia. Accordingly a need exists for methods of studying such turbulence both quantitatively and qualitatively.

Numerous schemes for meeting this need have been proposed and investigated heretofore, only one of which, the hot wire anemometer, satisfies at all well the aerodynamical requirements. This device, ostensibly simple and relatively satisfactory, consists of a fine wire heated electrically by current flow and subjected to the variable cooling induced by the velocity fluctuations in the moving air stream. The corresponding variations in voltage drop of the wire are amplified to measurable proportions for oscillographic study or direct meter indication.

\section{DIFFICULTIES INHERENT IN \\ Hot Wire ANemometer Scheme}

Inherent in this scheme are 2 fundamental difficulties, compensation and calibration, both of which are surmountable but not without the introduction of some ambiguity into the results. Since ordinary turbulence has a frequency spectrum much like that of acoustic noise, compensation is essential because the hot wire responses much greater for low frequency velocity fluctuations than for those of high frequency. In order to obtain something approximating a faithful reproduction of turbulence a distorting circuit must be employed in the amplifying equipment, the characteristics of which are dictated by the various factors contributing to the "lag" of the wire. The lag characteristics of the wire are, in turn, determined from a theoretical analysis necessarily involving assumptions and approximations. Such procedure is a rational one and necessary; but, it is unfortunate that no standard turbulence exists nor has any velocity fluctuation thus far been devised whose characteristics, in magnitude and quality, are known without serious ambiguity to serve as an over-all check on the fidelity of the hot wire apparatus except for very low frequencies. Moreover, the fairly obvious test which may be made of compensation by passing through the wire a heat- 
ag current containing an alternating component of known frequency gives only a partial verification ince conditions under which the wire operates for this measurement are not equivalent to those encountered in actual use as an anemometer. Then too, this compensation necessarily influences calibration, that is, affects the interpretation of amplifier output measurements in terms of actual or relative velocity fluctuation. The method of computing magnitudes of turbulence from hot wire observations is, of course, not relevant to the present paper. Significant, however, is the fact that compensation and calibration are both functions of average air speed, a circumstance which increases both the experimental work and the subsequent reduction of data.

\section{These Difficulties Not Encountered IN THE Glow Discharge Anemometer}

Thus, despite the many desirable attributes of the hot wire anemometer and the excellent work which it has made possible, the thermal lag of the wire introduces difficulties which have stimulated a search for other means of studying turbulence. This quest has led to a new electrical method employing as its sensitive element a minute glow discharge. Such an electrical discharge at atmospheric pressure is characterized principally by a cathode glow a few thousandths of a centimeter in length and potential of the order of 300 volts, and a positive column having a voltage gradient of approximately 1,500 volts per centimeter. The corresponding discharge current is in the range of from 10 to $30 \mathrm{ma}$. The total voltage of such a glow, about 400 volts, acted on by a transverse stream of air, was found to increase substantially with in-

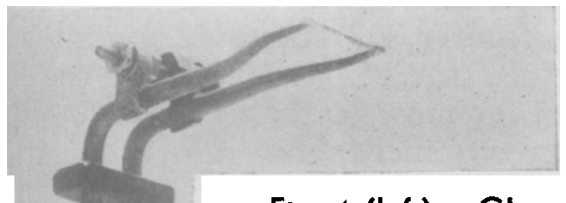

Fig. 1 (left). Glow anemometer mounting

Fig. 3 (right). Electrical circuit of glow and measuring apparatus

Fig. 2 (below). Glow anemometer with adjustable gap creasing air velocity, throughout a range in speed as great as that ordinarily employed in wind tunnel studies. Moreover, this glow is responsive to turbulence and gives results which are in excellent agreement with those obtained with the hot wire. A description of these results and the essential apparatus yielding them, together with a criticism of the glow discharge method of analyzing turbulence, form the substance of this presentation.

\section{Apparatus of the Glow Discharge Anemometer}

A few exploratory experiments served definitely to establish the essential fact that a glow discharge is sensitive to air velocity. Moreover, these qualitative tests indicated practical limits for the length of the glow and magnitude of the discharge current, which, together with aerodynamical requirements as to size and disposition of the discharge electrodes specified the essential apparatus and the electrical quantities involved.

A mounting of electrodes for practical work is shown in Fig. 1. The electrodes proper are silversoldered to the supporting rods which are kept at proper spacing by the screw clamping device and terminate in an insulating block.

A more elaborate gap mounting employed to study the variation of glow voltage with length for several values of air velocity is indicated in Fig. 2. The illustration is self-explanatory, giving clearly the means of adjusting gap length as well as showing the section of glass rod which insulates one electrode from the rest of the assembly. The removable tapering tips which hold the electrode points make relatively simple a change of tip material.

The electrical circuit found to be most desirable appears in Fig. 3. A transformer and rectifier tube
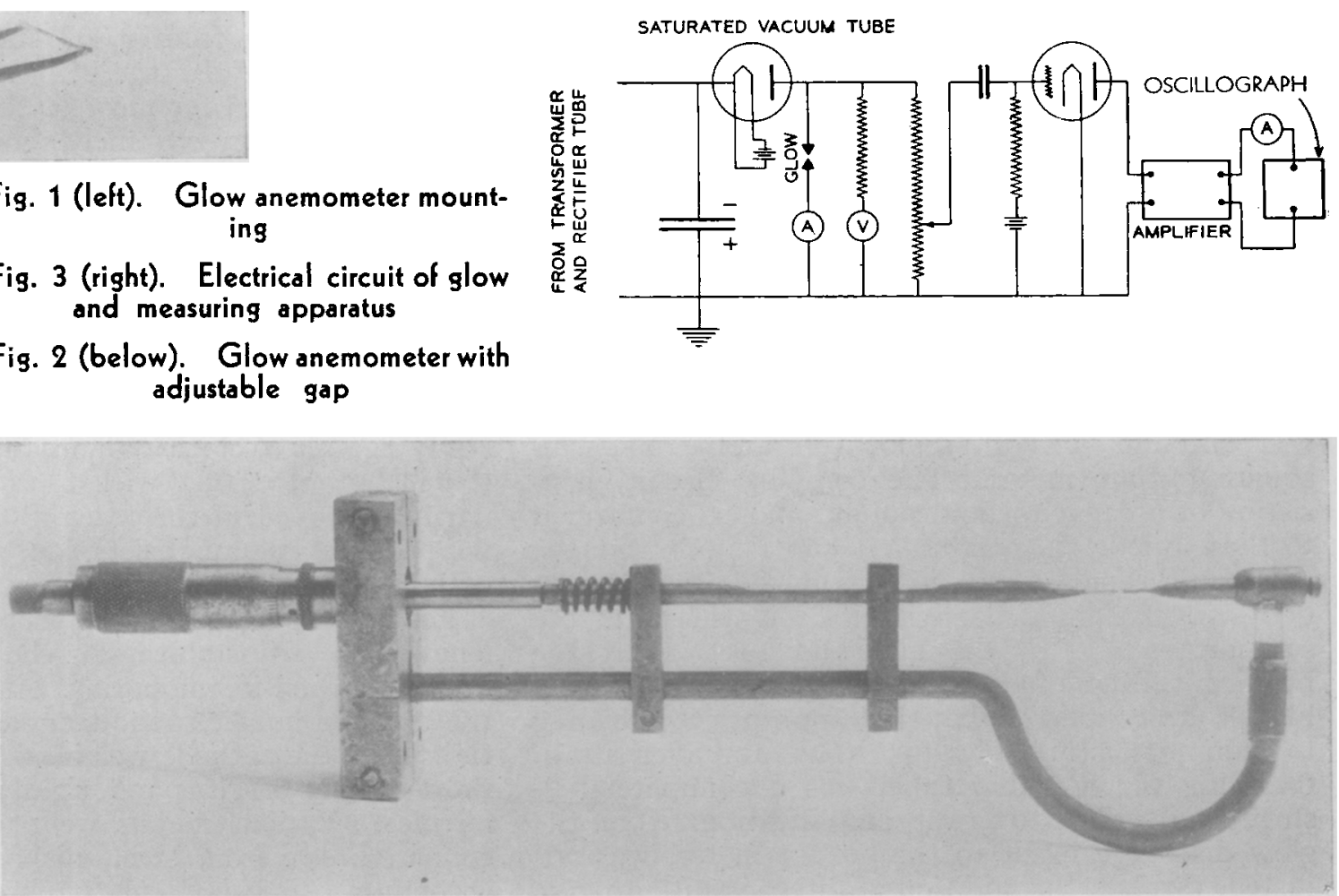


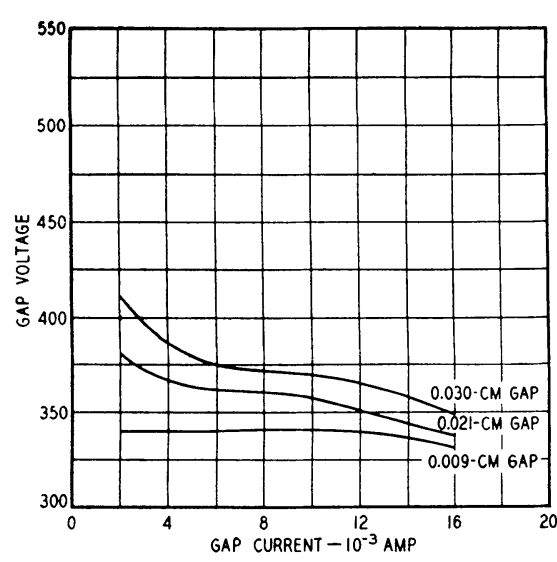

Fig. 4. Air velocity $=0$

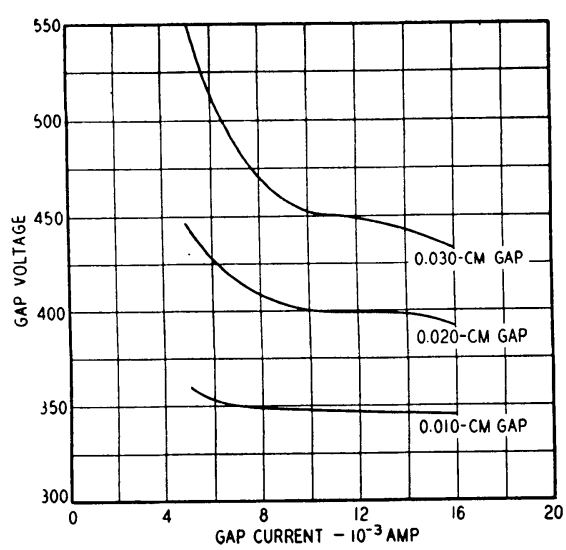

Fig. 5. Air velocity $=8$ meters per second Fig. 6. Air velocity $=20$ meters per second

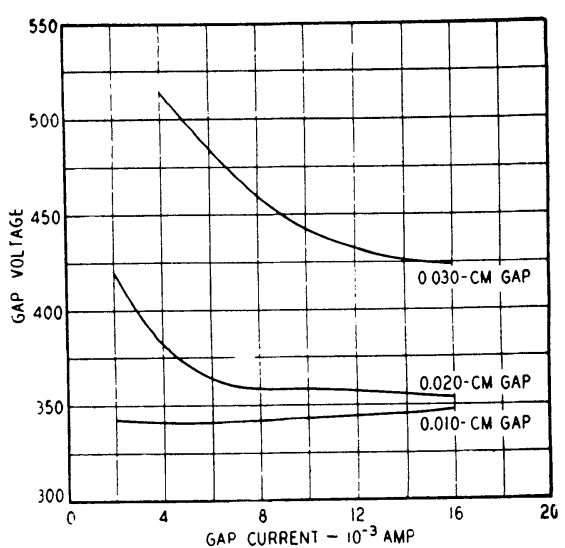

Figs. 4-6. Glow voltage-current relationships

maintained the capacitor at a potential of approximately 5,000 volts. The glow discharge current was held constant by the saturated vacuum tube shown and was adjustable through filament current in a range of from zero to $50 \mathrm{ma}$. (This circuit is essentially that employed by Phillips Thomas in his studies on the microphonic properties of a glow discharge. ${ }^{1}$ ) The change in average voltage of the glow was measured with a microammeter having a series resistor of approximately 14 megohms and with a bucking circuit (not shown) set to give zero meter indication for the discharge operating in still air. Accordingly with the latter arrangement the increase in glow voltage produced by the air appears as a direct meter indication. An over-all calibration of this potential measuring circuit was effected by inserting in series with the glow voltage, by an obvious switching arrangement, a 45 -volt battery which produced a meter deflection corresponding to this known increase in voltage. Such an arrangement was desirable inasmuch as frequent adjustment of the bucking circuit was necessitated by changes made in gap length or current.

Measurement of average air velocity was made with a standard pitot tube and precision manometer developed at the Guggenheim aeronautics laboratory of the California Institute of Technology, and when less accuracy was required, with a commercial air speed meter.

The foregoing equipment sufficed for an investigation of glow voltage as a function of air velocity, gap length, and discharge current. The fluctuations in voltage induced by turbulence in the moving air stream were transmitted through a coupling circuit and amplifier to an oscillograph and root-meansquare output meter. The coupling circuit, consisting of a capacitor and potentiometer, conformed to these important conditions: sufficient impedance to permit rapid fluctuation of glow voltage; adequate insulation in the capacitor to withstand the striking voltage of the gap and to prevent the running voltage of the glow from affecting the grid bias of the coupling tube; and appropriate shielding to minimize stray pick-up. The amplifier itself, following the coupling tube, was a commercial 2stage unit. The over-all amplification, from the glow discharge input to the output meter, was adjusted, through the addition of filter circuits, to give very closely a uniform frequency response from 50 to 2,500 cycles.

\section{Limits of Glow Current}

The limits for glow current are empirical: too low a value leads to a stringy type of spark discharge at high voltage; too high a value leads to excessive heating and burning of the cathode, rendering impossible any reproducibility in curves of gap voltage as a function of air velocity. Moreover, for a certain limited range of current values, the gap voltage is nearly independent of current-a happy circumstance which makes small fluctuations in emission current of the control tube insignificant.

\section{Details of Gap and Electrodes}

The length of the discharge, or more precisely the length of the gap, since the former is indefinite due to spread of the cathode glow, is an important factor in this work as will be seen in the data to follow. Lengths ranging from $0.002 \mathrm{~cm}$ to $0.200 \mathrm{~cm}$ were tested and a useful range of from $0.010 \mathrm{~cm}$ to 0.025 $\mathrm{cm}$ was employed for most of the work with turbulent air flow. An extremely short gap is insensitive to air velocity while a long discharge is blown downstream into a bow shape having a greatly lengthened positive column with corresponding abnormally high voltage. Moreover, this "blowing back" gives an unstable type of discharge, apparently in a transition state between the spark and the glow types of conduction, which "sings" at a high audible frequency and therefore is worthless as a device for studying air velocity fluctuations. Accordingly, it will be noted that with the lengths of gap ordinarily employed, of the order of $0.015 \mathrm{~cm}$, practically no positive column exists, only the characteristic cathode glows being visible.

For the investigation of the effect. of discharge length, the adjustable gap, shown in Fig. 2, was used. The entire mounting with the exception of the cathode holder, was at anode or ground potential, while the cathode was insulated therefrom by a short length of glass rod rigidly gripped in metal ferrules. Various metals were used for electrodes: copper, brass, iron, steel, nichrome, nickel, silver, chromium, tungsten, platinum, and platinum-iri- 
dium. All electrodes were approximately $0.15 \mathrm{~cm}$ in diameter with ends ground conically at about 20 deg taper. Of these various materials platinum, platinum-iridium, and tungsten were the most satisfactory, giving the minimum of difficulty from destruction of the points. Platinum gave a more consistently stable discharge as well as better reproducibility in data than did tungsten and therefore was used for the bulk of the tests.

In such a test device, the diameter of the electrodes should be comparable to the dimension of the glow itself in order that velocity, as measured, be definitely that of the stream flow and not the true velocity modified by the presence of bulky electrodes. Hence, only small diameter material may be used for cathode and anode with consequent small surface and cross section area for heat dissipation and conduction. Therefore, to minimize irregularities in behavior of the discharge due to heating of the electrodes a current value must be used which is as small as will give a satisfactory response of the gap over a particular velocity range.

\section{Tests Without Turbulence Introduced}

The first group of tests had as an object a study of the interdependence of glow voltage, glow current,

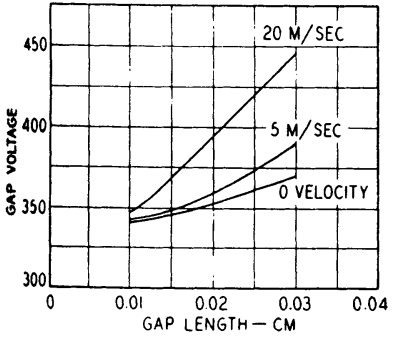

Fig. 7. Glow voltage as a function of sap length

Current $=10 \mathrm{ma}$

Air velocity $=0,5$, and 20 meters per second

gap length, and air velocity, the air flow being turbulent to a negligible extent. The procedure was to measure glow voltage as a function of current for specific values of air velocity and particular settings of gap length. Typical results of these measurements are given in Figs. 4, 5, and 6. Glow voltage is plotted against current, each figure being for a designated air velocity and each curve corresponding to the gap length indicated. In order to avoid confusion only 3 gap settings are given.

The nature of these curves is in accord with that of a normal glow discharge. The extremely short gap gives a glow voltage virtually independent of gap current, except for high air velocity. Indeed, one may say that the short glow is almost wholly a cathode phenomenon, and only when the discharge is effectively lengthened by the transverse air motion, does it exhibit the negative resistance quality so characteristic of the typical glow discharge. The longer gap, however, gives rise to a discharge having the familiar hyperbolic characteristic for all air ve- locities including zero. Yet, considering all of the curves with a view to finding an optimum current value for air flow study, one sees that for the velocity range of these data 10 or $15 \mathrm{ma}$ in the discharge gives an over-all minimum of voltage change due solely to current fluctuation.

Choosing, then, a particular value of current to be maintained in the discharge, curves may be derived from the preceding data which indicate the dependence of gap voltage on gap length, air velocity being an arbitrary parameter. Such curves, presented in Fig. 7, exhibit the essentially linear nature to be expected as the relationship between voltage and length of discharge, and suggest that the increase in glow voltage with velocity is due largely to a lengthening of the discharge.

Then, finally, from such data were obtained curves relating the essential quantities, voltage and air velocity. These curves, such as the one of Fig. 8 could be made to have either increasing or decreasing slope with higher air speed, giving sensitivity in either the low or the high velocity range by suitable choice of gap length and current.

These calibration curves indicate a voltage change of the order of volts per meter per second velocity increment, a response which is ample for investigating turbulence of quite small magnitude. Indeed, the sensitivity of the glow to turbulence, as predicted by such data, is approximately 100 times as great as that of the hot wire anemometer, substantially simplifying the amplifier problem. Accordingly it remained to demonstrate that the glow discharge responds faithfully to turbulence and to that only.

\section{Tests on Response to Turbulence}

That the glow really responds to turbulence was shown qualitatively in the wakes behind cylinders and flat plates where conditions are fairly well known from accepted theoretical and experimental aerodynamical research. ${ }^{3.5}$ Moreover, the oscillographic representation and the amplifier output meter readings observed during any traverse of the air flow downstream from an obstruction, conformed beautifully with results of similar tests made in air and other fluids by various means. The glow anemometer gave no indication of turbulence in those portions of the flow known to be steady, thus eliminating the possibility of microphonic response, but it responded unquestionably to the velocity fluctuations in regions of disturbed flow, bringing out, in addition

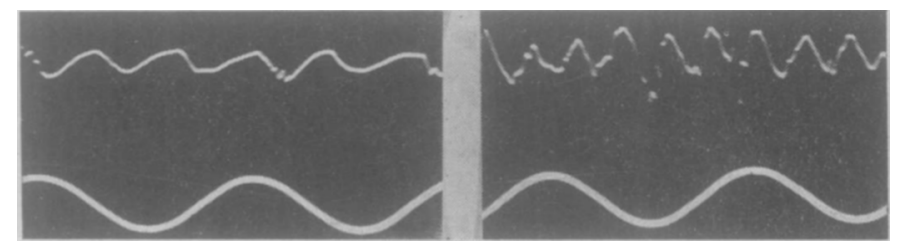

Fig. 9. Turbulence behind cylinder for 2 air velocities

Left, upper trace-9.9 meters per second velocity, frequency 133 cycles. Right, upper trace- 16.3 meters per second velocity, frequency 220 cycles Lower traces-50-cycle timing waves 
to the usual random variations, the more or less regular sequence of vortices so characteristic of the wake behind a regular body. The oscillograms of Fig. 9 represent such a vortex sequence behind a cylinder for different air velocities, with a 50-cycle timing wave included to establish the frequency of the turbulence. These frequencies were found to agree with values computed from the vortex theory of von Kármán ${ }^{3}$ which states that frequency is directly proportional to velocity and inversely proportional to the diameter of the cylinder. The factor of proportionality is 0.18 for any consistent set of units. ${ }^{4}$

Further confirmation of the response of the glow to turbulence came with simultaneous oscillograph records for hot wire and glow located at similar points in the 2-dimensional flow behind a cylinder.

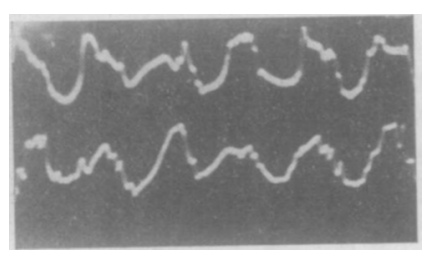

Fig. 10. Comparison between glow and compensated hot wire systems for measuring turbulence behind a cylinder

Upper trace. Hot wire scheme Lower trace. Glow discharse scheme

The oscillograms of Fig. 10 show clearly that the glow response is as nearly like that of the compensated hot wire as the instantaneous velocities at 2 points in a turbulent fluid would compare. Moreover, simultaneous quantitative measurements of turbulence, root-mean-square velocity fluctuation divided by average air speed, gave good agreement between glow and hot wire methods.

The calibration of the glow anemometer for quantitative measurements of turbulence was achieved in a relatively simple way. Either mean-square or root-mean-square velocity fluctuation is a convenient quantity for theoretical consideration of turbulence, and either is conveniently metered in the amplifier output. Accordingly, if at a certain average speed, turbulence produced a particular root-mean-square output current, that same value of current was later caused to flow by impressing an adjustable alternating voltage at approximately 500 cycles across the terminals of the glow discharge. For example, suppose that 3 volts effective, 500 cycles, at the gap produced the same value of output current as the turbulence. A voltage fluctuation of 3 volts rootmean-square therefore must have been caused by air speed variation. A portion of the curve of gap voltage against average air speed establishes the value of the slope $\frac{\Delta e}{\Delta v}$ at the test velocity-only 1 or 2 points on either side being necessary-and leads at once to a value of root-mean-square velocity fluctuation. In short, the calibration as used was of the over-all type in which all essential data were taken at the time of the turbulence measurements.

\section{Discussion of Results}

The data which have been obtained with the glow anemometer demonstrate that such an electri- cal discharge is sensitive to air velocity in both the steady and turbulent states. Moreover, for the study of air turbulence this electrical method has certain definite advantages over the hot wire scheme, notably in calibration and simplicity of amplifying equipment. Yet, the glow itself has one notably outstanding defect which, fortunately, is more of a nuisance than an evil. Any such electrical discharge will inevitably result in some slow loss of electrode material, through sputtering and chemical modifications in the presence of air. And, as a consequence, a particular length of gap as used in this work will lengthen appreciably during the course of a run of 15 or $20 \mathrm{~min}$ duration, so that final data will not coincide with the original average calibration curve. Still, this difficulty is more apparent than real for 2 reasons. First, in turbulence studies made in the course of usual wind tunnel or other air flow work, ample opportunity exists for obtaining average voltage-speed data near the operating point at a time near enough to that of the turbulence measurement to give the characteristic slope. And, second, the slopes of 2 calibration curves corresponding to slightly different gap lengths are, for the same average air speed, practically identical. Consequently this defect in the glow anemometer is not serious, it merely prescribes a technique of measurement. And, indeed, the hot wire, because of accidental adherence of dust particles or due to other surface charges, has been found to be erratic in calibration from day to day.

One further critical comment on the glow anemometer is pertinent. The thermal time lag of the hot wire is of first importance in turbulence investigations. The glow, on the contrary, appears to have no lag in its response to air velocity change. The evidence which can be cited in support of this statement, however inadequate, must be considered in the absence of a satisfactory method of experimental verification, as previously discussed. Nothing in the mechanism of an atmospheric glow of the dimensions used could introduce a time lag significant in the frequency range of turbulence, for ion mobilities are adequately high.

Then, too, in the work which employed a glow as a microphone ${ }^{1}$ it was found that a flat frequency response was approached as shorter gaps were employed, a fact which is significant when it is realized that for an anemometer a much shorter gap length was used than for a microphone. And, finally, the direct comparison of glow and compensated hot wire showed no indication of time lag in the glow discharge.

In conclusion, it may thus be stated that a new and interesting property of the atmospheric glow discharge - the variation of voltage with air velocity - has been shown to exist and that this property may be utilized in an anemometer for the study of turbulent air flow. Such an anemometer, the author believes, is a useful adjunct to the hot wire equipment, and, because of the essential ruggedness of the glow mounting, may be incorporated in a wind tunnel for routine measurements, an opinion which was encouraged largely by the consistent interest shown in this work by Dr. Theodor von 
Karman, director of the Guggenheim laboratory of aeronautics at the California Institute of Technology.

\section{REFERENCES}

1. A Diaphragmless Microphone for Radio Broadcasting, Phillips Thomas. A.I.E.E. Trans., v. 42, 1923, p. 1111-14.

2. Efpect of Turbulence in Wind Tunnel Measurements, H. L. Dryden and A. M. Kuethe. Nat'l. Advisory Committee on Aeronautics, 1930, Report No. 342 , p. $147-73$

3. Ü Ber den Mechanismus des Flùssigkeits- Und Luftwiderstandes, T. v. Kármán and H. Rubach. Physikalische Zeitschrift, v. 13, 1912, p. 49-59.

4. On the Frequency of the Eddies Generated by the Motion of Circular Cylinders Through a Fluid, E. F. Relf and L. F. G. Simmons. Philosophical Mag., v. 49, Feb. 1925, p. 509-11.

5. Hydrodyamics (a book), Horace Lamb. Cambridge University press, ed. 6,1932 , p. 208 et seq.

\section{Distance Relay Action During Oscillations}

\begin{abstract}
The behavior of distance relays during system oscillations is analyzed in this paper, and curves are presented for determining whether the oscillations are likely to cause relay operation. Different types of distance relays are considered and general methods of analyzing system conditions are discussed.
\end{abstract}

\author{
By \\ E. H. BANCKER \\ MEMBER A.I.E.E. \\ E. M. HUNTER \\ ASSOCIATE A.I.E.E.
}

General Elec. Co., Schenectady, N.Y.

\section{O}

PERATING experience with directional distance relays has demonstrated their ability to clear short circuits rapidly and reliably. However, a few instances have been reported in which apparently a circuit breaker was opened when portions of the system were subjected to a power oscillation or lost synchronism because of a fault. Within recent years much study has been devoted to the effect of relay characteristics upon system stability and it now appears desirable to consider

Full text of a paper recommended for publication by the A.I.E.E. committee power transmission and distribution, and discussed at the A.I.E.E. North Eastern District meeting Worcester Mass. May 16-18, 1934 . A.E.E. North for discussion at the A. I. E. Pacific Coast convention Salt Lake Sch, Uted Sept 3-7, 1934 Manuscript submitted Feb. 27, 1934: March 19, 1934. Not published in pamphlet form. the converse, namely, the effect of an unstable system upon the relays protecting the unfaulted portions.

The term "unstable" is here used to denote any condition of operation during which the rotors of the connected synchronous apparatus do not maintain a substantially constant angular relationship with each other. Under this classification there can be 2 kinds of instability; (1) one is a continuous, unidirectional angular departure of one or more rotors with respect to the others which results in loss of synchronism, slip, or out-of-step operation; (2) the other is a fluctuating change of relative rotor positions, termed oscillating, swinging, or surging, which eventually will damp down to a condition of stable equilibrium.

There is some diversity of opinion as to whether or not distance relays should open any circuit breakers during out-of-step operation, some engineers believing that the essential separation should be performed manually or automatically at a predetermined point in conformity with the load conditions, and others caring little where it occurs, provided no load is left without a source of power.

On the other hand, opinion is nearly unanimous in preferring that under oscillating conditions no circuit breaker shall be opened by any protective relay. Since such an eventuality is within the bounds of possibility, this paper is presented to point out the procedure to be followed in determining whether distance relays in a particular location are in any danger of tripping unnecessarily during swings. Methods of avoiding such false operation are presented which may be used should a study show a need for remedial measures.

\section{Types of Relays and Their Characteristics}

Two general types of directional distance relays are available, one using line reactance and the other line impedance as the measure of the distance to the point of fault. Both types have 3 distinct elements: (1) a directional unit; (2) an ohm unit or units consisting, in the reactance relay, of one or more reactance measuring devices, and in the impedance relay of several impedance measuring devices corresponding to the number of steps in the relay; and (3) a timing unit. In the impedance relay the indicated line impedance is obtained by comparing the magnitude of the voltage with that of the current. In the reactance relay the comparison is made between the current and the inductive component of the voltage.

Both types of these relays have a "step characteristic" giving definite operating times for different fault locations, as shown in Fig. 1. A fault in zone 1 (often called instantaneous zone), which covers approximately 80 per cent of the line distance to relay station 2 , is cleared in minimum time. A fault in zone 2, which covers the last 20 per cent of the line to relay station 2 and something less than 80 per cent of the line distance to station 3 , is cleared in intermediate time, and a fault in zone 3 is cleared in back-up time (so called because no relay should ever operate in this time unless some other device has failed to operate correctly). In considering 